\title{
Predicting sizes of hexagonal and gyroid metal nanostructures from liquid crystal templating
}

Article

Accepted Version

Asghar, K. A., Rowlands, D. A., Elliott, J. M. and Squires, A. M. (2015) Predicting sizes of hexagonal and gyroid metal nanostructures from liquid crystal templating. ACS Nano, 9 (11). pp. 10970-10978. ISSN 1936-086X doi:

https://doi.org/10.1021/acsnano.5b04176 Available at https://centaur.reading.ac.uk/46789/

It is advisable to refer to the publisher's version if you intend to cite from the work. See Guidance on citing.

To link to this article DOI: http://dx.doi.org/10.1021/acsnano.5b04176

Publisher: American Chemical Society

All outputs in CentAUR are protected by Intellectual Property Rights law, including copyright law. Copyright and IPR is retained by the creators or other copyright holders. Terms and conditions for use of this material are defined in the End User Agreement.

www.reading.ac.uk/centaur 
Central Archive at the University of Reading

Reading's research outputs online 


\title{
Predicting Sizes of Hexagonal and Gyroid Metal Nanostructures from Liquid Crystal Templating
}

\author{
Kaleem A. Asghar, ${ }^{\S, \dagger}$ Daniel A. Rowlands, ${ }^{\ddagger}$ Joanne M. Elliott, ${ }^{\dagger}$ and Adam M. Squires ${ }^{*,+}$ \\ ${ }^{\dagger}$ Department of Chemistry, University of Reading, Whiteknights Campus, Reading RG6 6AD, Berkshire, U.K., 'TCM Group, Cavendish Laboratory, \\ University of Cambridge, $19 \mathrm{~J} J$ Thomson Avenue, Cambridge CB3 OHE, U.K., and ${ }^{\S}$ Department of Chemistry, The University of Engineering and Technology, \\ Lahore 39021, Pakistan. All authors have given approval to the final version of the manuscript.
}

\begin{abstract}
We describe a method to predict and control the lattice parameters of hexagonal and gyroid mesoporous materials formed by liquid crystal templating. In the first part, we describe a geometric model with which the lattice parameters of different liquid crystal mesophases can be predicted as a function of their water/surfactant/oil volume fractions, based on certain geometric parameters relating to the constituent surfactant molecules. We demonstrate the application of this model to the lamellar $\left(L_{\alpha}\right)$, hexagonal $\left(H_{1}\right)$, and gyroid bicontinuous cubic $\left(V_{1}\right)$ mesophases formed by the binary Brij-56 $\left(\mathrm{C}_{16} \mathrm{EO}_{10}\right)$ /water system and the ternary Brij-56/hexadecane/water system. In this way, we demonstrate predictable and independent control over the size of the cylinders
\end{abstract}

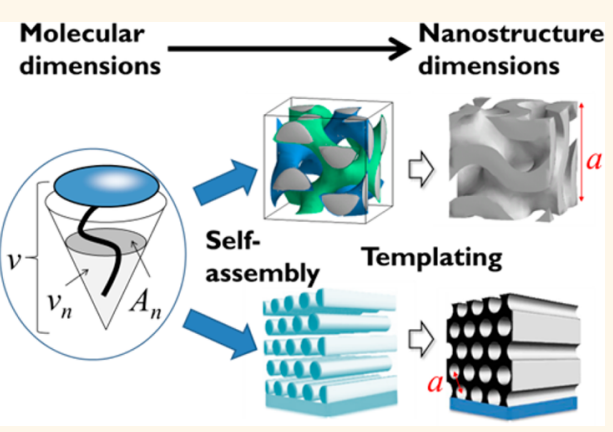
(with hexadecane) and their spacing (with water). In the second part, we produce mesoporous platinum using as templates hexagonal and gyroid phases with different compositions and show that in each case the symmetry and lattice parameter of the metal nanostructure faithfully replicate those of the liquid crystal template, which is itself in agreement with the model. This demonstrates a rational control over the geometry, size, and spacing of pores in a mesoporous metal.

KEYWORDS: mesoporous · liquid crystal templating · electrodeposition · gyroid

S urfactant-templated mesoporous materials, containing ordered periodic arrays of pores 2-50 $\mathrm{nm}$ in diameter, have had major impacts on chemical research and industries over the past few decades. The earliest and most widespread examples are mesoporous silica compounds such as the Mobil MCM family, which are used in heterogeneous catalysis in a range of applications $^{1}$ and which are typically produced by a hydrolysis reaction in the presence of the structure-directing surfactant.

Direct electrochemical templating of lyotropic liquid crystals has become an increasingly widespread alternative route to nanostructured materials. ${ }^{2}$ Its advantages are, first, that the electrochemical deposition process is flexible and can be applied to many different metals, ${ }^{2-6}$ metal oxides, ${ }^{7}$ and polymers ${ }^{8}$ and, second, that it can be carried out in a chemically mild, roomtemperature process. It is therefore compatible with a range of different conducting substrates, to which the approach provides good adherence of the mesoporous material. Importantly, as we show here, the nanostructure lattice parameter faithfully replicates that of the lyotropic liquid crystalline phase, which we can predict using the packing models we outline in this paper.

Direct electrochemical templating was originally applied to the $H_{1}$ phase (Figure 1) by Attard and co-workers in $1997^{2}$ to produce platinum films containing cylindrical mesopores. Since then, this phase has been used as a template for electrodeposition of a range of mesoporous materials to provide high surface area coatings with applications including catalysis, ${ }^{9}$ analysis, ${ }^{10,11}$ separation technology, ${ }^{12}$ optical devices, ${ }^{13}$ solar cells, ${ }^{14}$ and energy storage devices. ${ }^{15}$

Lyotropic liquid crystal systems also exhibit bicontinuous cubic phases, in particular, the gyroid phase (Figure 1). ${ }^{16}$ Mesoporous materials with this morphology have branching wires and pores with 3D symmetry ${ }^{17}$ and, therefore, have a number of advantages over the $1 \mathrm{D}$ hexagonal pore structure relating to *Address correspondence to
a.m.squires@reading.ac.uk.

Received for review July 7, 2015 and accepted October 23, 2015.

Published online

10.1021/acsnano.5b04176

C XXXX American Chemical Society 

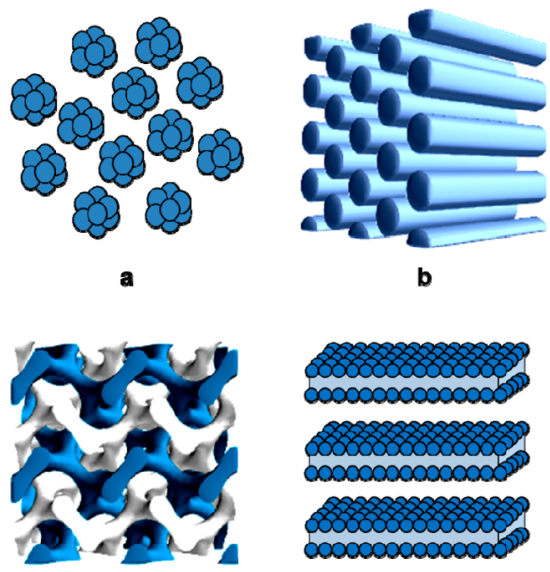

c

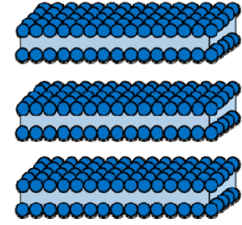

d

Figure 1. Lyotropic liquid crystalline phases: (a) micellar $\left(L_{1}\right)$, (b) hexagonal $\left(H_{1}\right),\left(\right.$ c) gyroid bicontinuous cubic $\left(V_{1}\right)$, and (d) lamellar $\left(L_{\alpha}\right)$.

diffusion and conductivity and overcoming problems with pore orientation and blockage $i^{18}$ moreover, it has been theoretically predicted that gyroid structures formed from low loss metals could act as negative refractive index metamaterials. ${ }^{19}$ Mesoporous materials with gyroid nanowire networks have found applications in actuators, ${ }^{20}$ photocatalysts, ${ }^{21}$ solar cells, ${ }^{22}$ electrodes, ${ }^{23}$ catalysts, ${ }^{24,25}$ and sensors. ${ }^{26}$ For the most part, these have not been directly templated from the liquid crystal phase (although we have recently reported the electrodeposition of a structurally related "single diamond" nanowire network using an inverse bicontinuous cubic phase). ${ }^{27}$ Instead, the templates used have in general been etched diblock copolymers ${ }^{24,28}$ or mesoporous silica that was itself chemically templated from the liquid crystal phase. ${ }^{25,26}$ However, some literature is available indicating that it is possible to form an inverted gyroid structure, containing 3D networks of pores, through direct liquid crystal templating, ${ }^{29}$ making a structure that is the inverse of the nanowire networks. This inverted form is expected to have similar properties and advantages in terms of its conductivity, diffusion, and mechanical integrity, but its formation through a chemically mild one-pot templating step makes it a more attractive synthetic route.

A number of physical properties are affected by the lattice parameter and nanostructure of mesoporous materials. These include diffusion, ${ }^{30-32}$ capacitance, $^{33,34}$ and catalyst stability. ${ }^{35,36}$ It is therefore desirable to be able to control the nanoarchitecture dimensions, typically achieved by varying the template used. There have been a number of attempts to develop models capable of predicting the nanoarchitecture of the lyotropic liquid crystalline phase and relating it to the size and shape of the constituent surfactant molecules. The original work, by Israelachvili et al., ${ }^{37}$ involves the surfactants' "packing parameter".
This has been used to describe micellar shape (cylindrical, spherical micelles, bilayer, etc.) and therefore provide a qualitative description of the resultant structure on templating. ${ }^{38}$ However, the model does not provide a quantitative prediction of, for example, lattice parameter or micelle size. In any case, for mesoporous silica and materials produced through similar routes, substantial structural change occurs during solid formation: ${ }^{38}$ the packing parameter is affected by significant interactions between silica precursor and surfactant; and the structure evolves on silica polymerization and subsequent calcination. Therefore, while general trends in the final silica nanostructure can be explained and controlled, the approach cannot provide a quantitative prediction of the final lattice parameter adopted.

Instead, researchers have employed empirical, qualitative approaches to control the nanoarchitecture. In some cases, they simply used different sized surfactant molecules ${ }^{4}$ or block copolymers. ${ }^{39-41}$ Continuous tunability in mesophase lattice parameter cannot be obtained easily in this way, and a range of slightly different molecules needs to be synthesized. In other cases, the composition of the soft template was varied by adding additional molecules, for example, hydrophobic liquids ${ }^{2}$ or cosurfactants. ${ }^{42}$ In this paper, we build on these largely empirical approaches, and construct a theoretical framework leading to a quantitative geometric model that can be used to predict nanoarchitecture dimensions. We outline a derivation of this framework, and demonstrate how we can use it to produce predictably controllable nanomaterials with different geometries, exploiting the faithful replication of liquid crystal nanostructure dimensions that electrochemical deposition allows.

\section{MODEL}

We assume that the surfactant molecule is incompressible and, therefore, that the molecular volume has a constant value, $v$. Within different mesophases the surfactant molecules occupy different average molecular shapes. In a flat lamellar structure, the molecule occupies a cylindrical geometry, with a constant crosssection along the length of the molecule. As we form different mesophases with increasingly curved interfaces from lamellar through gyroid and hexagonal to micellar, we would expect the area per molecule to increase toward the headgroup region and water interface, and decrease toward the hydrophobic chains, as shown in Figure 2. Our model assumes that somewhere in between there is a position on the molecule where the molecular area does not change with different curvatures. We call this the "Area Neutral Surface" and use $A_{n}$ and $v_{n}$ to denote the crosssectional area per molecule at this surface, and the volume per molecule on the hydrophobic side of this surface. This naming convention follows equivalent 


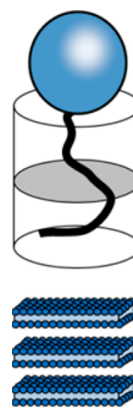

a
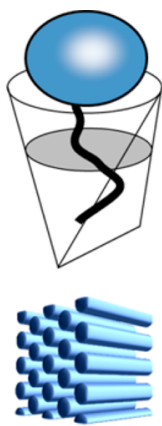

b
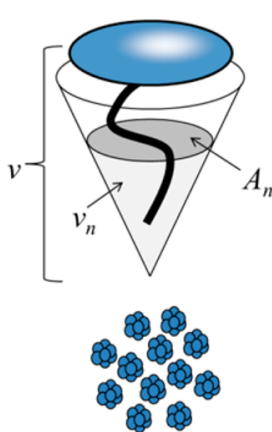

C
Figure 2. Surfactant aggregate structures (bottom row) and corresponding average molecular shapes (top row) for (a) lamellar, (b) hexagonal, and (c) micellar phases, illustrating the parameters $A_{n}, v_{n}$, and $v$. These represent, respectively, the area per molecule of the area neutral surface (represented as a gray disk), the volume per molecule below this surface, and the volume of the molecule.

analysis that has been carried out on type II lipids. ${ }^{43}$ The three geometric parameters that characterize the surfactant molecule, and which we assume are constant for all phases and lattice parameters adopted by the surfactant, are $A_{n}, v_{n}$, and the total molecular volume $v$.

Given these molecular parameters, the lattice parameters can be predicted as a function of surfactant volume fraction, $\phi_{\text {surf }}$ for each phase as outlined below. A more detailed derivation is given in the Supporting Information.

For the fluid lamellar $L_{\alpha}$ phase, we assume that there is no chain interdigitation and, therefore, that the bilayer thickness is twice the length of the surfactant molecule. We assume that, on changing the surfactant/ water ratio, the bilayers move further apart or closer together, while their thickness does not change. The lattice parameter, $a$, is the sum of the thicknesses of the bilayer and the water layer, and depends on $\phi_{\text {surf, }}$ the surfactant volume fraction, as follows:

$$
a=\frac{2 v}{A_{n} \phi_{\text {surf }}}
$$

The hexagonal $H_{1}$ phase consists of surfactant cylinders whose radius can be determined from the molecular parameters (see Figure 3). The derivation is outlined in the Supporting Information. Again, we assume that decreasing the water/surfactant ratio and therefore increasing $\phi_{\text {surf }}$ simply moves these cylinders closer together. This gives

$$
a=\sqrt{\frac{8 \pi v_{n} v}{\sqrt{3} A_{n}^{2} \phi_{\text {surf }}}}
$$

For the gyroid bicontinuous cubic $\left(V_{1}\right)$ phase, we assume that the area neutral surfaces lie parallel to the surface that passes through the middle of the water region, which is a mathematical shape known as a (triply periodic) minimal surface. (The surfactant-water

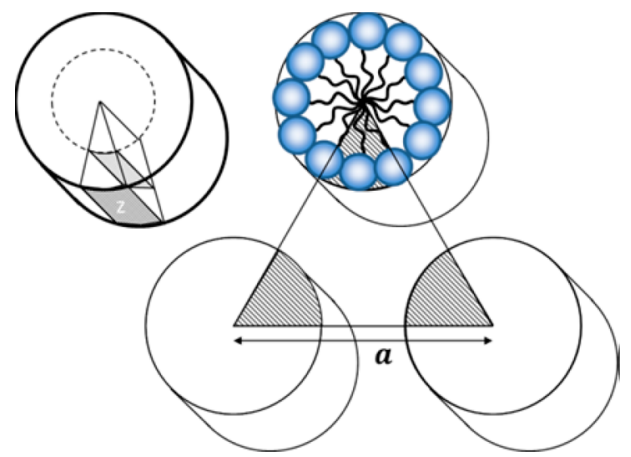

Figure 3. Schematic showing the surfactant-water interface (solid line circles) and area neutral surface (dotted line circle) in a hexagonal phase of lattice parameter $a$. The wedge shows approximate molecular dimensions.

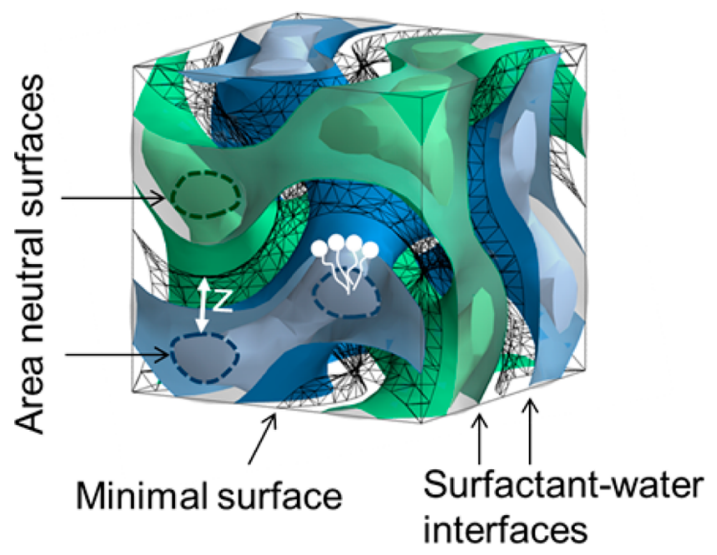

Figure 4. Schematic diagram of a gyroid bicontinuous cubic phase. The area neutral surfaces lie a distance $z$ away from the minimal surface, which is shown as a net.

interfaces also form parallel surfaces.) We consider the two area neutral surfaces to lie a distance $z$ away from the minimal surface, one on either side (see Figure 4).

In a given bicontinuous cubic phase, we assume that $z$ remains constant over the surface. However, for different samples with different surfactant volume fractions and different lattice parameters, we allow $z$ to change; the molecular parameters $v, v_{n}$ and $A_{n}$ remain constant. An analogous system exists in the inverse bicontinuous cubic phases, where the minimal surface passes through the center of a surfactant bilayer; these structures have been analyzed by Templer, employing a parallel interface model similar to the one we adopt here. ${ }^{44}$ Using an analogous treatment, outlined in more detail in the Supporting Information, we can find the values of $z$ and $\phi_{\text {surf }}$ as functions of the lattice parameter $a$ by finding solutions to

$\frac{2 \sigma_{0} a^{2}}{A_{n}}\left(1+\frac{2 \pi \chi}{\sigma_{0} a^{2}} z^{2}\right)=\frac{\left(a^{3}-2 \sigma_{0} a^{2} z\left(1+\frac{2 \pi \chi}{3 \sigma_{0} a^{2}} z^{2}\right)\right)}{v_{n}}=\frac{a^{3} \phi_{\text {surf }}}{v}$

where $\chi$ and $\sigma_{0}$ are the Euler characteristic and the dimensionless area per unit cell, taking values of -8 and 3.091 , respectively, for the gyroid minimal surface. ${ }^{43}$ 
For the addition of a hydrophobic liquid such as hexadecane in the hexagonal phase, we assume that this liquid does not mix with the hydrophobic tails of the surfactant but rather forms a discrete cylinder within the surfactant cylinder. Such behavior is more likely to occur when using longer chain hydrophobic liquids, such as hexadecane. ${ }^{45}$ Based on this assumption, our model keeps $A_{n}$ constant and effectively allows $v$ and $v_{n}$ to increase by an amount equivalent to the volume of hexadecane per molecule of surfactant, which is equal to $\left(\phi_{\text {oil }} / \phi_{\text {surf }}\right) v$ where $\phi_{\text {oil }}$ is the volume fraction of hydrophobic liquid. This gives

$$
a=\sqrt{\frac{8 \pi\left(v_{n}+v \frac{\phi_{\text {oil }}}{\phi_{\text {surf }}}\right)\left(v+v \frac{\phi_{\text {oil }}}{\phi_{\text {surf }}}\right)}{\sqrt{3} A_{n}^{2}\left(\phi_{\text {surf }}+\phi_{\text {oil }}\right)}}
$$

In this paper, we apply the models described above to the surfactant Brij-56 (C16EO10). We show how data for lattice parameter vs composition can be used to determine the parameters $A_{n}$ and $v_{n}$ for this molecule and demonstrate how this information can be used to predict the geometry, size and spacing of pores in the gyroid and hexagonal phases by varying composition in binary (surfactant/water) and ternary (surfactant/ water/hexadecane) mixtures. We then use the model to predict the spacing of mesoporous platinum films electrodeposited from binary and ternary templating phases with both hexagonal and gyroid structures.

\section{RESULTS AND DISCUSSION}

Swelling Behavior and Determination of Molecular Parameters. The lattice parameters of binary mixtures of the surfactant Brij-56 and $\mathrm{H}_{2} \mathrm{O}$ are shown in Figure $5 \mathrm{a}$ as a function of the surfactant volume fraction, $\phi_{\text {surf }}=$ $\phi_{\text {Brij-56. The expected general decrease in lattice }}$ parameter with increasing $\phi_{\text {surf }}$ can be seen. The data were obtained at different temperatures. The model assumes that $A_{n}, v_{n}$, and $v$ are independent of temperature; the evidence for this is that the lattice parameter values within a particular phase do not change significantly with temperature (Figure SI3).

These data were used to estimate the molecular parameters, as follows.

First, the molecular volume is estimated from the density of the pure surfactant and its molecular weight using

$$
v=\frac{M_{\mathrm{w}}}{\rho N_{\mathrm{A}}}
$$

Here, $M_{w}$ is the molecular weight, $\rho$ is the density, and $N_{\mathrm{A}}$ is Avogadro's number.

For Brij-56, we use values of $M_{\mathrm{w}}=683 \mathrm{~g} / \mathrm{mol}$ and the value quoted earlier of $\rho=0.977 \mathrm{~g} / \mathrm{cm}^{3}$ (SigmaAldrich) from which we estimate $v=1.16 \times 10^{-21} \mathrm{~cm}^{3}=$ $1160 \AA^{3}$.
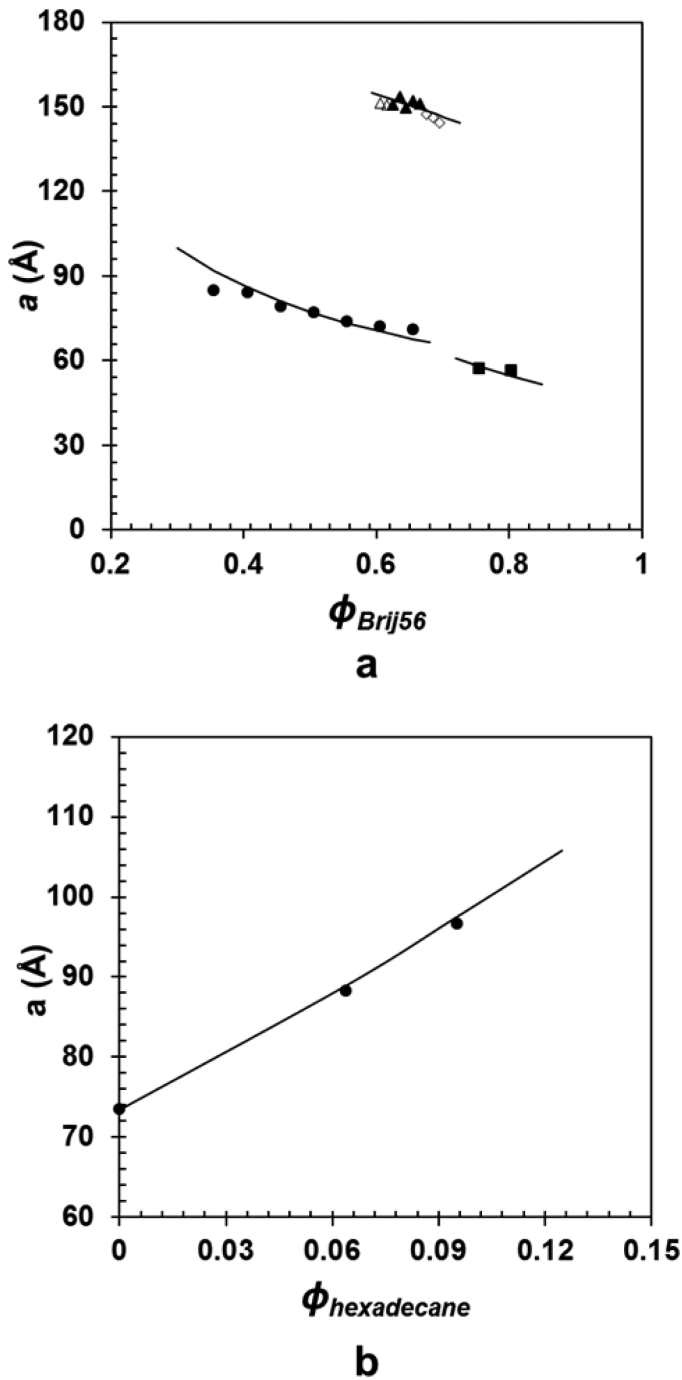

Figure 5. (a) Lattice parameters (a) as a function of surfactant volume fraction $\phi_{\text {Brij-56 }}$ for the hexagonal $\left(H_{1}\right)$ (solid circles), gyroid $\left(V_{1}\right)$ (empty triangles, solid triangles and empty diamonds), and lamellar $\left(L_{\mathrm{a}}\right)$ (solid squares) phases from binary mixtures. (b) Lattice parameters $(a)$ as a function of $\phi_{\text {hexadecane }}$ for $H_{1}$ phases from ternary mixtures for constant weight $\%$ water of $45\left(\phi \mathrm{H}_{2} \mathrm{O}=0.44\right)$. The SAXS data were collected at 35 and $45^{\circ} \mathrm{C}$ for the $H_{1}$ and the $L_{\alpha}$ phases, respectively, while for the $V_{1}$ phases they were collected at $55{ }^{\circ} \mathrm{C}$ (open triangles), $50{ }^{\circ} \mathrm{C}$ (solid triangles), and $45{ }^{\circ} \mathrm{C}$ (open diamonds). Solid lines show theoretical curves for $A_{n}=54 \AA^{2}, v_{n}=500 \AA^{3}$.

$A_{\mathrm{n}}$ is estimated from the lattice parameter $a(57 \AA)$ of the fluid lamellar phase of surfactant volume fraction $\phi_{\text {surf }}=\phi_{\text {Brij-56 }}=0.754$ using

$$
A_{n}=\frac{2 v}{a \phi_{\text {surf }}}
$$

This gives $A_{n}=54 \pm 2 \AA^{2}$.

$v_{n}$ is determined using the data for the hexagonal phase and the values of $v$ and $A_{n}$ above.

$$
\phi_{\text {surf }}=\frac{8 \pi}{\sqrt{3}} \frac{v_{n} v}{\left(A_{n} a\right)^{2}}
$$

From this we obtain $v_{n}=500 \pm 40 \AA^{3}$. 
These parameters were then used to fit all of the data for the $L_{\alpha}, H_{1}$, and $V_{1}$ phases, as shown by the solid lines in Figure 5.

Given that $v$ was determined independently, the $L_{\alpha}$ and $H_{1}$ data were effectively modeled with one independent parameter each $\left(A_{n}, v_{n}\right)$. The $V_{1}$ data were then predicted from these with no further independent variables, representing a very good agreement.

Figure $5 \mathrm{~b}$ shows the lattice parameter data for $\mathrm{H}_{1}$ phases of the ternary system Brij-56/hexadecane $/ \mathrm{H}_{2} \mathrm{O}$, varying $\phi_{\text {hexadecane. }}$ Again, the theoretical model gives an extremely good fit to the experimental data, and again, we stress that the theoretical values were predicted using only the values of $A_{n}, v_{n}$, and $v$ obtained previously with no further fitting of independent variables. This suggests validity of the assumptions implicit in our model.

Conservation of Structure under Electrochemical Deposition Conditions. We now go on to demonstrate that there is no significant change in lattice parameter on replacement of water with hexachloroplatinic acid (HCPA), the electrolyte solution used for electrodeposition of platinum, nor on subsequent electrodeposition of platinum.

Figures 6 and 7 show data for the $H_{1}$ phase on varying the water content (Figure 6) or adding hexadecane (Figure 7), while Figure 8 shows data for the $V_{1}$ phase and then in each case upon replacing water with HCPA and then templating with SAXS patterns as shown below in each case.

Figures $6 a$ and 8a show the lattice parameters of different binary mixtures of Brij-56 and HCPA in the $H_{1}$ phase (Figure 6) and $V_{1}$ phase (Figure 8 ), varying the surfactant volume fraction, $\phi_{\text {brij; }}$ Figure $7 a$ shows the lattice parameters of ternary mixtures of Brij-56/ hexadecane/HCPA in the $H_{1}$ phase on addition of hexadecane. The data, shown as open circles, are superimposed on the corresponding swelling data for mixtures containing water (instead of HCPA); the water data, shown as solid circles, are also shown in Figure 5 . The data show very good agreement in each case; as Brij-56 is a nonionic polyoxyethylene surfactant, its headgroup is not significantly affected by changes in $\mathrm{pH}$ or ionic strength.

Figures $6 \mathrm{~b}, 7 \mathrm{~b}$ and $8 \mathrm{~b}$ show the 1D SAXS patterns for different mesoporous hexagonal $\left(H_{1}-\mathrm{ePt}\right)$ and gyroid $\left(V_{1}-\mathrm{ePt}\right)$ platinum films overlaid on those of their respective Brij-56/HCPA and Brij-56/hexadecane/HCPA templating mixtures for several different compositions. The positions of the Bragg peaks match in each case, demonstrating a match in the mesoporous structure and unit cell size before and after templating. The structures are characterized by reflections with relative positions in the ratio $1: \sqrt{ } 3$ for hexagonal ${ }^{46}$ and $\sqrt{ } 6: \sqrt{ } 8$ for gyroid. ${ }^{47}$

The relative peak intensities in the templated metal films vary and are in some cases different from those of
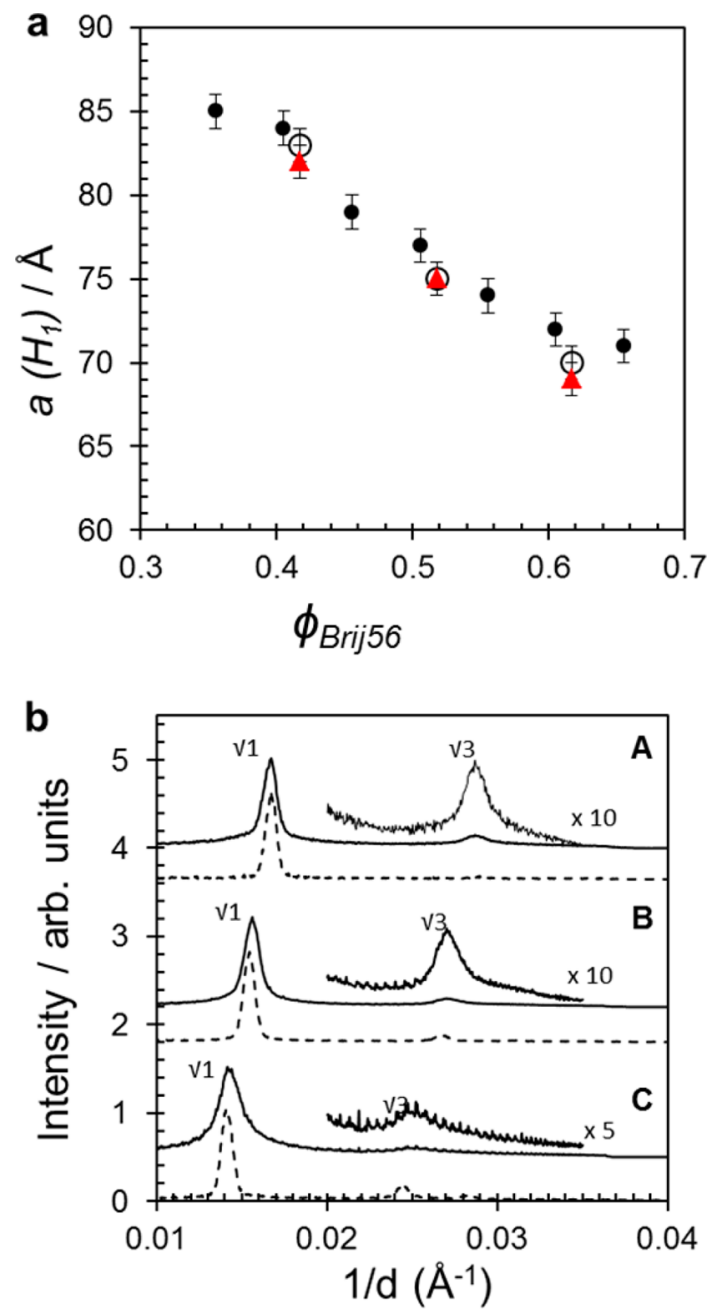

Figure 6. (a) Lattice parameter (a) as a function of $\phi_{\mathrm{Brij}-56}$ in binary mixtures of Brij $56 / \mathrm{H}_{2} \mathrm{O}$ (solid circles) and of Brij 56/HCPA (open circles) for the $H_{1}$ phases and the $H_{1}$-ePt films from the corresponding HCPA mixtures (red solid triangles). (b) Overlaid SAXS patterns for the $\left(H_{1}\right.$-ePt) films (solid lines) templated from the mixtures (dotted lines) of $\phi_{\text {Brij-56 }} / \phi_{\text {HCPA }}=0.61: 38(\mathrm{~A}), 0.51: 0.48$ (B), and 0.41:0.58 (C). SAXS data for the hexagonal phases were collected at $35^{\circ} \mathrm{C}$.

the templating mixture. This could be due to a combination of changes in electron density and its distribution throughout the unit cell and orientation in the metal film relative to the substrate. ${ }^{48}$ In the gyroid material, the peaks are also broader, probably reflecting less long-range order; thus, the $\sqrt{ } 8$ reflection appears as a shoulder in the SAXS patterns from $V_{1}$-ePt (Figure $8 \mathrm{a}$ ). It is interesting to note that a similar phenomenon was observed in the templating of inverse bicontinuous cubic phases, ${ }^{27}$ whereas in contrast the $H_{1}$-ePt films are much better ordered. We cannot at present suggest a definitive explanation for this difference, but assume that it reflects the greater resistance of the $H_{1}$-ePt structure to distortion, perhaps due to its containing a continuous metallic layer in a plane perpendicular to the cylindrical pores.

Finally, the lattice parameters for the mesoporous metals have been plotted on Figures $6 a, 7 a$, and $8 a$ as a 

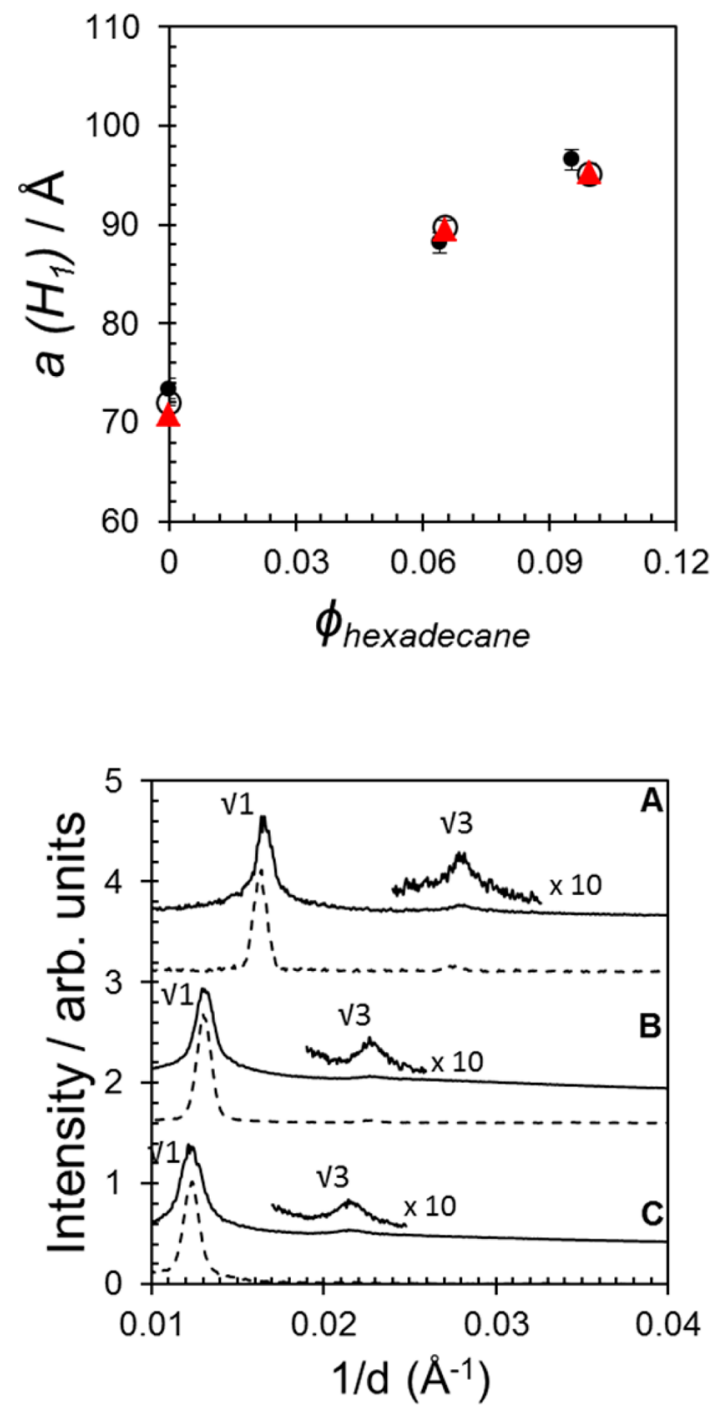

Figure 7. (a) Lattice parameter (a) as a function of $\phi_{\text {hexadecaner }}$ for $\phi_{\text {hexadecane }} / \phi_{\text {Brij-56 }} / \phi \mathrm{H}_{2} \mathrm{O}=0: 0.56: 0.44,0.064: 0.49: 0.44$ and $0.10: 0.46: 0.43$ (solid circles) and $\phi_{\text {hexadecane }} / \phi_{\text {Brij-56 }} / \phi_{\mathrm{HCPA}}=$ 0:0.57:0.43, 0.065:0.50:0.43 and 0.10:0.46:0.43 (empty circles) for the $H_{1}$ phases and the $H_{1}$-ePt films templated from the corresponding HCPA mixture (red solid triangles). (b) Overlaid SAXS patterns for the $\left(H_{1}\right.$-ePt) films (solid lines) templated from the mixtures (dotted lines) of $\phi_{\text {hexadecane }}$ / $\phi_{\text {Brij-56 }} / \phi_{\mathrm{HCPA}}=0: 0.57: 0.43(\mathrm{~A}), 0.065: 0.50: 0.43$ (B), and $0.10: 0.46: 0.43$ (C). SAXS data for the hexagonal phases were collected at $35^{\circ} \mathrm{C}$.

function of the surfactant volume fraction of the template phase (solid triangles). The general agreement confirms that the nanodimensions of the metal in each case reflects that of the precursor lyotropic liquid crystalline phase, which itself can be described by our model, as shown in Figure 5.

Assumptions and Extensions to Model. Our model is based on the assumption that $A_{n}$ and $v_{n}$ are constant for a certain surfactant molecule across different phases with different aggregate nanostructures; in other words, it makes the assumption of the existence of an area neutral position within the molecule. It makes no assumptions about the location in the
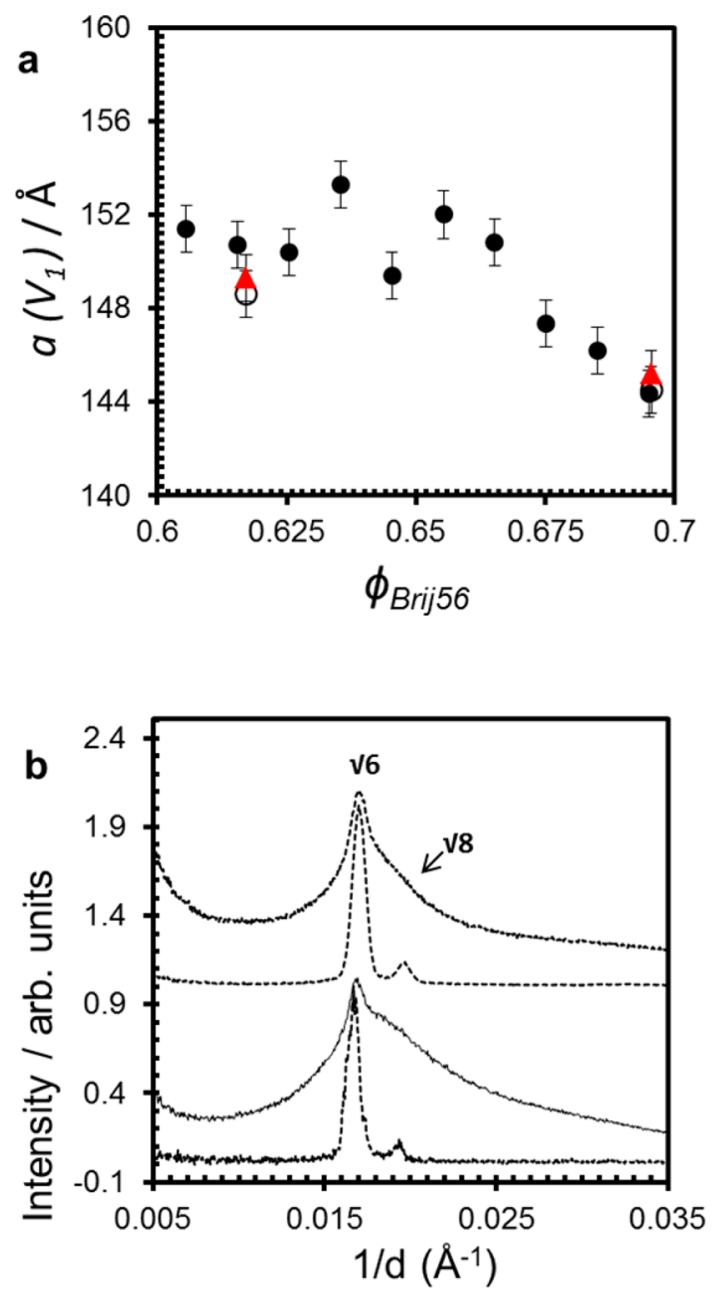

Figure 8. (a) Gyroid $\left(V_{1}\right)$ lattice parameter (a) as a function of surfactant volume fraction $\phi_{\mathrm{Brij}-56}$ in binary mixtures of Brij-56/ $\mathrm{H}_{2} \mathrm{O}$ (solid circles; data points also shown in Figure 5a), Brij-56/HCPA (empty circles), and $V_{1}$-ePt films (red solid triangles). (b) Overlaid SAXS patterns for the $V_{1}$-ePt films (solid lines) templated from the mixtures (dotted lines) of $\left(\phi_{\text {Brij-56 }} / \phi_{\mathrm{HCPA}}\right)=(0.70: 30)$ (top) and (0.62:0.38) (bottom).

molecule of this position. In this section we discuss these implications further.

Analyzing other polyoxyethylene surfactant systems, Kunieda and co-workers ${ }^{48}$ found that molecular area "changes very slightly with the surfactant concentration...if the type of liquid crystal [i.e. phase] is unchanged" and in particular "In the $\mathrm{L}_{\alpha}$ phase [area per molecule] slightly increases with increasing water content, but it becomes almost constant in the hexagonal phase." In our own data, the fluid lamellar phase also shows a change in molecular area with surfactant volume fraction: the value of $A_{n}=54 \AA^{2}$ was obtained from the lower surfactant content data point $\left(\phi_{\text {Brij-56 }}=\right.$ 0.754), while the second data point $\left(\phi_{\text {Brij-56 }}=0.80\right)$ gives a value of $A_{n}=51 \AA^{2}$. The results can be rationalized by supposing that in the lamellar phase at higher surfactant volume fractions the polyoxyethylene headgroups are close enough to interact and 
distort one another, thereby changing the bilayer thickness and area per molecule. We have therefore chosen to model our data with the value obtained at lower surfactant volume fractions within the lamellar phase, where headgroup interactions between adjacent bilayers are minimized. Using this as the basis for our model, the remainder of the lattice parameter data for the other phases can be modeled reasonably well. It is possible that a better fit could be obtained by allowing the ratio $v_{n} / A_{n}$ to vary for the hexagonal phase or for $v_{n}$ and $A_{n}$ to take on different values for the gyroid cubic phase. However, we felt that such an approach would require the addition of further fitting parameters, with less obvious physical meaning, and reduce the predictive power of the model.

In our analysis so far, we have made no assumptions about the physical location within the molecule of the area neutral position and, therefore, attached no physical significance to $v_{n}$. Intuitively, we might expect the area neutral position to lie at the interface between the hydrophobic and hydrophilic parts of the molecule and $v_{n}$ to therefore represent the volume of the hydrophobic chain, and we test this hypothesis here. Different approaches have been explored to estimate the volume of the hydrophilic and hydrophobic parts of polyoxyethylene surfactants; for a general surfactant $\mathrm{CmEOn}$ where the hydrophilic headgroup has the molecular formula $\left[\left[\mathrm{OCH}_{2} \mathrm{CH}_{2}\right]_{n} \mathrm{OH}\right.$, Sakya and coworkers ${ }^{49}$ suggest that its volume can be estimated as $(60.9 n+17) \AA^{3}$. For the surfactant in this paper, Brij-56 (C16EO10), where $n=10$, this gives a value of $626 \AA^{3}$. Our experimentally determined value for the volume of molecule on the hydrophilic side of the neutral surface is $v-v_{n}=1160-500=660 \pm 40 \AA^{3}$. This agreement within experimental error indicates that the area neutral surface does indeed approximately lie at the boundary between the hydrophilic and hydrophobic parts of the molecule. To further test the generality of this observation and the robustness of our approach, we have analyzed data from a second surfactant, $\mathrm{C} 12 \mathrm{EO} 6$ (chemical formula $\mathrm{C}_{12} \mathrm{H}_{25}\left[\mathrm{OCH}_{2} \mathrm{CH}_{2}\right]_{6} \mathrm{OH}$ ), whose estimated headgroup volume using Sakya's expression is $382 \AA^{3}$. Again, our data can be fit well using a single set of parameters $v=765 \AA^{3}$ (from molecular weight and density), $A_{n}=50 \pm 2 \AA^{2}$ and $v_{n}=390 \pm 10 \AA^{3}$ (see the Supporting Information, Figure SI5). The part of the molecule on the hydrophilic side of the area neutral surface therefore has volume $v-v_{n}=765-390=375 \pm$ $10 \AA^{3}$, again agreeing with the estimated headgroup volume. (We also investigated an alternative approach using equivalent molecular parameters to estimate the volume of the hydrophobic chain. For a chain of formula $\mathrm{C}_{m} \mathrm{H}_{2 m+1}$ Malcolmson and Laurence ${ }^{50}$ estimate the volume to be $(27(m-1)+55) \AA^{3}$. For C16EO10 and C12EO6 this respectively gives values of 460 and $352 \AA^{3}$; our corresponding values for $v_{n}$ are
$500 \pm 40$ and $390 \pm 10 \AA^{3}$. The agreement is therefore not as good as that obtained by estimating the hydrophilic headgroup volume.)

The molecular area at the neutral surface, $A_{n}$, does not have such a straightforward relationship with molecular formula. Malcolmson and Lawrence ${ }^{50}$ have studied a range of polyoxyethylene surfactants and reported a linear relationship between the area per molecule $a_{0}\left(\AA^{2}\right)$ and the molecular weight of the hydrophilic head, represented by $n$, the number of $\left[\mathrm{OCH}_{2} \mathrm{CH}_{2}\right]$ units, through the relationship $a_{0}=1.56 n+$ 33.32. For the surfactants $\mathrm{C} 16 \mathrm{EO} 10$ and C12EO6 $(n=10$ and $n=6$ ), this would suggest values of $a_{0}=48.9 \AA^{2}$ and $a_{0}=42.7 \AA^{2}$, respectively. Our experimental data give values of $A_{n}=54 \pm 2 \AA^{2}$ and $A_{n}=50 \pm 2 \AA^{2}$, respectively. Thus, although they show the same qualitative trend, this does not yet represent a suitable quantitative estimate for $A_{n}$.

So far we have only discussed polyoxyethylene surfactants. For other nonionic systems, different methods would be required to estimate hydrophilic headgroup and hydrophobic chain volumes, to estimate $v_{n}$; indeed, it is possible that for some surfactant systems the area neutral surface would no longer lie at the interface between the two (for example, for type II liquid crystals formed by biological lipids, it was found to lie between carbon atoms 1 and 2 of the hydrocarbon chain ${ }^{43}$ ). On extending further to ionic systems, we would expect even more assumptions in our model to break down. Our model assumes that bilayers in the lamellar phase and cylinders in the hexagonal phase have no interaction and therefore maintain their dimensions on changing water content, only moving further apart or closer together. In ionic systems, there are significant electrostatic interactions, and quantities such as bilayer thickness and micelle cylinder radius change significantly with surfactant volume fraction. ${ }^{51}$ The assumption of constant $A_{n}$ would almost certainly provide a less satisfactory model for the structures adopted by the lyotropic liquid crystalline template. Furthermore, ionic systems are more sensitive to the ionic strength and $\mathrm{pH}$ of the aqueous medium. Since the metal electrodeposition reaction produces dramatic changes in these parameters locally to the electrode surface, we would expect the further result that the nanostructure of the templated metal differs significantly from that of the template prior to deposition.

\section{CONCLUSIONS}

We have demonstrated a model that predictably describes how controlling the volume fraction of hexadecane and Brij-56 determines the dimensions of the hexagonal $H_{1}$ and the gyroid $V_{1}$ lattices in the respective lyotropic phases. We have further demonstrated the structures of the templated hexagonal $\left(H_{1}-\mathrm{ePt}\right)$ and gyroid $\left(V_{1}-\mathrm{ePt}\right)$ films retained 
the structures of their corresponding templates. Taken together, this suggests for the first time a quantitative rational design of nanostructure in mesoporous metals.

\section{MATERIALS AND METHODS}

The surfactant polyoxyethylene (10) cetyl ether (commonly known as (16EO10, Brij-56, or Brij (10), hexachloroplatinic acid (HCPA, $0.2 \mathrm{M}, 99.9 \%)$, and hexadecane were purchased from Sigma-Aldrich and were used as received. Pure water (prepared by passing deionized, distilled water through a Milli-Q water purification system) was used throughout the experiments. The full list of templating mixtures is given in the Supporting Information (section $\mathrm{C}$ ). A range of liquid crystalline mixtures between 35 and 65 wt \% compositions of Brij- $56 / \mathrm{H}_{2} \mathrm{O}$ and two mixtures of (50:45:5) and (47.5:45.0:7.5) wt \% composition of Brij-56/ $\mathrm{H}_{2} \mathrm{O} /$ hexadecane were prepared to investigate the phase behavior of the hexagonal $\left(H_{1}\right)$ phases. For the preparation of the mesoporous $\left(H_{1}\right.$-ePt) films, mixtures of $40,50,55$, and 60 wt \% compositions of Brij-56/HCPA and (50:45:5) and (47.5:45:7.5) wt \% composition of Brij-56/ $\mathrm{H}_{2} \mathrm{O} /$ hexadecane were prepared, and their phase behavior was also investigated before templating. Similarly, for the phase-behavior studies of bicontinuous cubic $\left(V_{1}\right)$ phases, a range of mixtures from 60 to $69 \mathrm{wt} \%$ compositions of Brij- $56 / \mathrm{H}_{2} \mathrm{O}$ and 60 and $68 \mathrm{wt} \%$ of Brij-56/HCPA were prepared. Mixtures of 75 and 80 wt \% composition of Brij-56/ $\mathrm{H}_{2} \mathrm{O}$ were prepared for the investigation of lamellar phases. The different wt $\%$ composition of the liquid crystalline mixtures for the hexagonal, the bicontinuous cubic, and the lamellar phases were then converted to the volume fraction of each component $\phi x_{i}$ using the formula

$$
\phi_{x_{i}}=\frac{m\left(x_{i}\right) / \rho\left(x_{i}\right)}{\sum_{j} m\left(x_{j}\right) / \rho\left(x_{j}\right)}
$$

where $m\left(x_{i}\right)$ and $\rho\left(x_{i}\right)$ represent, respectively, the mass and density of the component $x_{i}$. The values for the densities used in these calculations were as follows: $0.977 \mathrm{~g} / \mathrm{mL}$ for the Brij-56, $1.05 \mathrm{~g} / \mathrm{mL}$ for the HCPA solution, and $0.773 \mathrm{~g} / \mathrm{mL}$ for hexadecane (values obtained from MSDS information, Sigma-Aldrich).

The hexagonal, bicontinuous cubic, and lamellar phase mixtures were investigated using a Bruker AXS Nanostar system fitted with a two-dimensional (2-D) detector. The Nanostar system was equipped with a compact 3-D pinhole collimation system which can form a well-defined spot size of approxi-

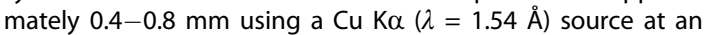
energy of $45 \mathrm{kV}$. The temperature-controlled measurements for the different liquid crystalline phases were done on an mri Physikalische Geräte $\mathrm{GmbH}$ high-temperature heating stage; the liquid crystalline mixtures were heated between 20 and $80^{\circ} \mathrm{C}$ at a ramp rate of $3^{\circ} \mathrm{min}^{-1}$. The $2 \mathrm{D}$ SAXS patterns were then integrated and analyzed with ImageJ software using "YAX integration" macros developed in house. ${ }^{52}$

All of the mesoporous Pt films from the $H_{1}$ and the $V_{1}$ phases were electrochemically deposited at $-0.1 \mathrm{~V}$ vs silver/silver chloride in a conventional three-electrode electrochemical cell. Large area $\left(0.2-0.5 \mathrm{~cm}^{2}\right)$ working electrodes were cut from a gold DVD, with the plastic coating peeled off and the exposed gold layer ( $\sim 10-12 \mathrm{~nm}$ thick) used as the depositing substrate. A flag-shaped piece of platinum was used as the counter electrode and a silver/silver chloride (determined to be $+0.248 \mathrm{~V}$ vs a normal hydrogen electrode) was used as the reference electrode. The deposition of the $\left(H_{1}\right.$-ePt) films from different compositions of Brij-56/HCPA and Brij-56/hexadecane/HCPA was carried out at $35 \pm 1{ }^{\circ} \mathrm{C}$. The deposition of $\left(V_{1}-\mathrm{ePt}\right)$ films was carried out at $40 \pm 1$ and $55 \pm 1^{\circ} \mathrm{C}$ from the $68 \mathrm{wt} \%$ Brij-56/ HCPA and $60 \mathrm{wt} \%$ Brij-56/HCPA mixtures, respectively. After the deposition experiments, the deposited $\left(H_{1}-\mathrm{ePt}\right)$ and $(\mathrm{V} 1-\mathrm{ePt})$ films were kept in deionized water for $24 \mathrm{~h}$ to remove the surfactant. After washing, the mesoporous $\left(H_{1}-\mathrm{ePt}\right)$ and $\left(V_{1}-\mathrm{ePt}\right)$ films were characterized using a Bruker AXS Nanostar system at camera lengths of 67 and $107 \mathrm{~cm}$. TEM images were also obtained from the $\left(H_{1}-\mathrm{ePt}\right)$ and $\left(V_{1}-\mathrm{ePt}\right)$ films using a Philips
CM20 transmission electron microscope, operated at $200 \mathrm{kV}$ (images shown in Supporting Information, Figure SI4). For TEM analysis the nanostructured platinum film was scraped off the substrate using a scalpel and placed on a copper grid with supporting carbon film (300 mesh).

Conflict of Interest: The authors declare no competing financial interest.

Supporting Information Available: The Supporting Information is available free of charge on the ACS Publications website at DOI: $10.1021 /$ acsnano.5b04176.

Derivation of structural relations; experimental data for lattice parameter as a function of temperature; compositions of mixtures used; TEM Images, and lattice parameter vs surfactant content data and theoretical fits for a second surfactant C12EO6 (PDF)

Acknowledgment. We thank the Department of Chemistry, University of Engineering and Technology Lahore, Pakistan, for providing funding for K.A.A. The project was further funded by EPSRC Grant EP/F036566. In addition, we thank the University of Reading Chemical Analysis Facility (CAF) and the Centre for Advanced Microscopy for providing access to instrumentation.

\section{REFERENCES AND NOTES}

1. Ying, J. Y.; Mehnert, C. P.; Wong, M. S. Synthesis and Applications of Supramolecular-Templated Mesoporous Materials. Angew. Chem., Int. Ed. 1999, 38 (1-2), 56-77.

2. Attard, G. S.; Bartlett, P. N.; Coleman, N. R. B.; Elliott, J. M.; Owen, J. R.; Wang, J. H. Mesoporous Platinum Films from Lyotropic Liquid Crystalline Phases. Science (Washington, DC, U. S.) 1997, 278 (5339), 838-840.

3. Attard, G. S.; Corker, J. M.; Göltner, C. G.; Henke, S.; Templer, R. H. Liquid-Crystal Templates for Nanostructured Metals. Angew. Chem., Int. Ed. Engl. 1997, 36 (12), 1315-1317.

4. Whitehead, A. H.; Elliott, J. M.; Owen, J. R.; Attard, G. S. Electrodeposition of Mesoporous Tin Films. Chem. Commun. 1999, 331-332.

5. Elliott, J. M.; Birkin, P. R.; Bartlett, P. N.; Attard, G. S. Platinum Microelectrodes with Unique High Surface Areas. Langmuir 1999, 15 (22), 7411-7415.

6. Bartlett, P. N.; Marwan, J. The Effect of Surface Species on the Rate of H Sorption into Nanostructured Pd. Phys. Chem. Chem. Phys. 2004, 6 (11), 2895.

7. Luo, H.; Zhang, J.; Yan, Y. Electrochemical Deposition of Mesoporous Crystalline Oxide Semiconductor Films from Lyotropic Liquid Crystalline Phases. Chem. Mater. 2003, 15 (20), 3769-3773.

8. Bender, F.; Chilcott, T. C.; Coster, H. G. L.; Hibbert, D. B.; Gooding, J. J. Characterisation of Mesoporous Polymer Films Deposited Using Lyotropic Liquid Crystal Templating. Electrochim. Acta 2007, 52 (7), 2640-2648.

9. Chen, A.; Holt-Hindle, P. Platinum-Based Nanostructured Materials: Synthesis, Properties, and Applications. Chem. Rev. 2010, 110 (6), 3767-3804.

10. Evans, S. A. G.; Elliott, J. M.; Andrews, L. M.; Bartlett, P. N.; Doyle, P. J.; Denuault, G. Detection of Hydrogen Peroxide at Mesoporous Platinum Microelectrodes. Anal. Chem. 2002, 74 (6), 1322-1326.

11. Imokawa, T.; Williams, K.-J.; Denuault, G. Fabrication and Characterization of Nanostructured Pd Hydride pH Microelectrodes. Anal. Chem. 2006, 78 (1), 265-271.

12. Yamaguchi, A.; Uejo, F.; Yoda, T.; Uchida, T.; Tanamura, Y.; Yamashita, T.; Teramae, N. Self-Assembly of a SilicaSurfactant Nanocomposite in a Porous Alumina Membrane. Nat. Mater. 2004, 3 (5), 337-341.

13. Miyata, H.; Fukushima, Y.; Okamoto, K.; Takahashi, M.; Watanabe, M.; Kubo, W.; Komoto, A.; Kitamura, S.; Kanno, Y.; 
Kuroda, K. Remarkable Birefringence in a TiO2-SiO2 Composite Film with an Aligned Mesoporous Structure. J. Am. Chem. Soc. 2011, 133 (34), 13539-13544.

14. Corma, A.; Atienzar, P.; Garcia, H.; Chane-Ching, J.-Y. Hierarchically Mesostructured Doped $\mathrm{CeO} 2$ with Potential for Solar-Cell Use. Nat. Mater. 2004, 3 (6), 394-397.

15. Orilall, M. C.; Wiesner, U. Block Copolymer Based Composition and Morphology Control in Nanostructured Hybrid Materials for Energy Conversion and Storage: Solar Cells, Batteries, and Fuel Cells. Chem. Soc. Rev. 2011, 40 (2), 520-535.

16. Seddon, J. M.; Templer, R. H. Cubic Phases of SelfAssembled Amphiphilic Aggregates. Philos. Trans. R. Soc., A 1993, 344 (1672), 377-401.

17. Holyst, R. Liquid Crystals: Infinite Networks of Surfaces. Nat. Mater. 2005, 4 (7), 510-511.

18. Landry, C. C.; Tolbert, S. H.; Gallis, K. W.; Monnier, a.; Stucky, G. D.; Norby, P.; Hanson, J. C. Phase Transformations in Mesostructured Silica/surfactant Composites. Mechanisms for Change and Applications to Materials Synthesis. Chem. Mater. 2001, 13 (6), 1600-1608.

19. Hur, K.; Francescato, Y.; Giannini, V.; Maier, S. a.; Hennig, R. G.; Wiesner, U. Three-Dimensionally Isotropic Negative Refractive Index Materials from Block Copolymer Self-Assembled Chiral Gyroid Networks. Angew. Chem., Int. Ed. 2011, 50, 11985-11989.

20. Vukovic, I.; Punzhin, S.; Vukovic, Z.; Onck, P.; De Hosson, J. T. M.; ten Brinke, G.; Loos, K. Supramolecular Route to Well-Ordered Metal Nanofoams. ACS Nano 2011, 5 (8), 6339-6348.

21. Sun, S.; Wang, W.; Zeng, S.; Shang, M.; Zhang, L. Preparation of Ordered Mesoporous Ag/WO3 and Its Highly Efficient Degradation of Acetaldehyde under Visible-Light Irradiation. J. Hazard. Mater. 2010, 178 (1-3), 427-433.

22. Crossland, E. J. W.; Kamperman, M.; Nedelcu, M.; Ducati, C.; Wiesner, U.; Smilgies, D. M.; Toombes, G. E. S.; Hillmyer, M. A.; Ludwigs, S.; Steiner, U.; et al. A Bicontinuous Double Gyroid Hybrid Solar Cell. Nano Lett. 2009, 9 (8), 2807-2812.

23. Alam, M. A.; Pimparkar, N.; Ray, B. The Future of Microelectronics Is...Macroelectronics. In Future Trends in Microelectronics; John Wiley \& Sons, Inc., 2010; pp 365-376.

24. Cheng, C.-F.; Hsueh, H.-Y.; Lai, C.-H.; Pan, C.-J.; Hwang, B.-J.; $\mathrm{Hu}, \mathrm{C} .-\mathrm{C} . ; \mathrm{Ho}, \mathrm{R} . \mathrm{M}$. Nanoporous Gyroid Platinum with High Catalytic Activity from Block Copolymer Templates via Electroless Plating. NPG Asia Mater. 2015, 7 (4), e170.

25. Kibsgaard, J.; Gorlin, Y.; Chen, Z.; Jaramillo, T. F. MesoStructured Platinum Thin Films: Active and Stable Electrocatalysts for the Oxygen Reduction Reaction. J. Am. Chem. Soc. 2012, 134, 7758-7765.

26. Urade, V. N.; Wei, T. C.; Tate, M. P.; Kowalski, J. D.; Hillhouse, H. W. Nanofabrication of Double-Gyroid Thin Films. Chem. Mater. 2007, 19 (11), 768-777.

27. Akbar, S.; Elliott, J. M.; Rittman, M.; Squires, A. M. Facile Production of Ordered 3D Platinum Nanowire Networks with "Single Diamond" Bicontinuous Cubic Morphology. Adv. Mater. 2013, 25, 1160-1164.

28. Crossland, E. J. W.; Kamperman, M.; Nedelcu, M.; Ducati, C.; Wiesner, U.; Smilgies, D. M.; Toombes, G. E. S.; Hillmyer, M. A.; Ludwigs, S.; Steiner, U.; et al. A Bicontinuous Double Gyroid Hybrid Solar Cell. Nano Lett. 2009, 9, 2807-2812.

29. Elliott, J. M.; Attard, G. S.; Bartlett, P. N.; Owen, J. R.; Ryan, N.; Singh, G. A Generic Approach to Electrodes with Novel Controllable Continuous Nanoarchitectures. J. New Mater. Electrochem. Syst. 1999, 2 (4), 239-241.

30. Negrini, R.; Mezzenga, R. Diffusion, Molecular Separation, and Drug Delivery from Lipid Mesophases with Tunable Water Channels. Langmuir 2012, 28, 16455-16462.

31. Vallet-Regí, M.; Balas, F.; Arcos, D. Mesoporous Materials for Drug Delivery. Angew. Chem., Int. Ed. 2007, 46 (40), 75487558.

32. Das, S. K.; Kapoor, S.; Yamada, H.; Bhattacharyya, A. J. Effects of Surface Acidity and Pore Size of Mesoporous Alumina on Degree of Loading and Controlled Release of Ibuprofen. Microporous Mesoporous Mater. 2009, 118 (1-3), 267-272.
33. Esterle, T. F.; Sun, D.; Roberts, M. R.; Bartlett, P. N.; Owen, J. R. Evidence for Enhanced Capacitance and Restricted Motion of an lonic Liquid Confined in $2 \mathrm{~nm}$ Diameter Pt Mesopores. Phys. Chem. Chem. Phys. 2012, 14 (11), 3872-3881.

34. Chmiola, J.; Yushin, G.; Dash, R.; Gogotsi, Y. Effect of Pore Size and Surface Area of Carbide Derived Carbons on Specific Capacitance. J. Power Sources 2006, 158 (1), 765-772.

35. Rauber, M.; Alber, I.; Muöller, S.; Neumann, R.; Picht, O.; Roth, C.; Schoökel, A.; Toimil-Molares, M. E.; Ensinger, W. Highly-Ordered Supportless Three-Dimensional Nanowire Networks with Tunable Complexity and Interwire Connectivity for Device Integration. Nano Lett. 2011, 11, 2304-2310.

36. Krins, N.; Bass, J. D.; Grosso, D.; Henrist, C.; Delaigle, R.; Gaigneaux, E. M.; Cloots, R.; Vertruyen, B.; Sanchez, C. NbVO5Mesoporous Thin Films by Evaporation Induced Micelles Packing: Pore Size Dependence of the Mechanical Stability upon Thermal Treatment and Li Insertion/ Extraction. Chem. Mater. 2011, 23 (18), 4124-4131.

37. Israelachvili, J. N.; Mitchell, D. J.; Ninham, B. W. Theory of Self-Assembly of Hydrocarbon Amphiphiles into Micelles and Bilayers. J. Chem. Soc., Faraday Trans. 2 1976, 72, 1525.

38. Huo, Q.; Margolese, D. I.; Stucky, G. D. Surfactant Control of Phases in the Synthesis of Mesoporous Silica-Based Materials. Chem. Mater. 1996, 8 (5), 1147-1160.

39. El-Safty, S. A.; Hanaoka, T.; Mizukami, F. Large-Scale Design of Cubic la3d Mesoporous Silica Monoliths with High Order, Controlled Pores, and Hydrothermal Stability. Adv. Mater. 2005, 17 (1), 47-53.

40. Zhao, D.; Feng, J.; Huo, Q.; Melosh, N.; Fredrickson, G. H.; Chmelka, B. F.; Stucky, G. D. Triblock Copolymer Syntheses of Mesoporous Silica with Periodic 50 to 300 Angstrom Pores. Science (Washington, DC, U. S.) 1998, 279 (5350), 548-552.

41. Soler-Illia, G. J. A. A.; Crepaldi, E. L.; Grosso, D.; Sanchez, C. Designed Synthesis of Large-Pore Mesoporous SilicaZirconia Thin Films with High Mixing Degree and Tunable Cubic or 2D-Hexagonal Mesostructure. J. Mater. Chem. 2004, 14 (12), 1879-1886.

42. Ramos, L.; Ligoure, C. Copolymer-Induced Stabilizing Effect of Highly Swollen Hexagonal Mesophases. Langmuir 2008, 24 (10), 5221-5224.

43. Templer, R. On the Area Neutral Surface of Inverse Bicontinuous Cubic Phases of Lyotropic Liquid Crystals. Langmuir 1995, 11 (9), 334-340.

44. Templer, R. H.; Seddon, J. M.; Warrender, N. A.; Syrykh, A.; Huang, Z.; Winter, R.; Erbes, J. Inverse Bicontinuous Cubic Phases in 2:1 Fatty Acid/Phosphatidylcholine Mixtures. The Effects of Chain Length, Hydration, and Temperature. J. Phys. Chem. B 1998, 102 (97), 7251-7261.

45. Blin, J. L.; Otjacques, C.; Herrier, G.; Su, B.-L. Pore Size Engineering of Mesoporous Silicas Using Decane as Expander. Langmuir 2000, 16 (9), 4229-4236.

46. Sanchez, C.; Boissière, C.; Grosso, D.; Laberty, C.; Nicole, L. Design, Synthesis, and Properties of Inorganic and Hybrid Thin Films Having Periodically Organized Nanoporosity + . Chem. Mater. 2008, 20 (3), 682-737.

47. Squires, A.; Templer, R. H.; Ces, O.; Gabke, A.; Woenckhaus, J.; Seddon, J. M.; Winter, R. Kinetics of Lyotropic Phase Transitions Involving the Inverse Bicontinuous Cubic Phases. Langmuir 2000, 16, 3578-3582.

48. Kunieda, H.; Kabir, H.; Aramaki, K.; Shigeta, K. Phase Behavior of Mixed Polyoxyethylene-Type Nonionic Surfactants in Water. J. Mol. Liq. 2001, 90 (1-3), 157-166.

49. Sakya, P.; Seddon, J. M.; Templer, R. H.; Mirkin, R. J.; Tiddy, G. J. T. Micellar Cubic Phases and Their Structural Relationships: The Nonionic Surfactant System C12EO12/Water. Langmuir 1997, 13 (14), 3706-3714.

50. Malcolmson, C.; Lawrence, M. J. Three-Component NonIonic Oil-in-Water Microemulsions Using Polyoxyethylene Ether Surfactants. Colloids Surf., B 1995, 4 (2), 97-109.

51. Parsegian, V. A. Theory of Liquid-Crystal Phase Transitions in Lipid + Water Systems. Trans. Faraday Soc. 1966, 62, 848.

52. Gras, S. L.; Squires, A. M. Dried and Hydrated X-Ray Scattering Analysis of Amyloid Fibrils. Methods Mol. Biol. 2011, 752, 147-163. 